\title{
On the Measurement of Roller Skew of Tapered Roller Bearings
}

\author{
A Falodi ${ }^{1,2, a},{ }^{*} Y K_{\text {Chen }}^{3, b}, M^{2}$ Caspall ${ }^{1, c}, B$ Earthrowl ${ }^{1}$ and D Dell ${ }^{3}$ \\ ${ }^{1}$ Cooper Roller Bearings Company Ltd, Wisbech Road, Kings Lynn, Nolfork. PE30 5JX. UK \\ ${ }^{2}$ Lombard Medical Technologies PLC, 4 Trident Park, Oxfordshire OX11 7HJ, UK \\ ${ }^{3}$ University of Hertfordshire, Hatfield, Hertfordshire. AL10 9AB. UK \\ a abiodun_falodi@hotmail.com, b'Y.K.Chen@herts.ac.uk, ${ }^{c}$ mcaspall@kaydon.com \\ * Corresponding author
}

\section{Keywords: tapered roller bearing, roller skew, rolling element}

\begin{abstract}
Roller skew in roller bearings can cause heat generation and reduce bearing life. Therefore, design to minimise its occurrence is essential in bearing development. This study investigated the roller skew of a double row tapered roller bearing under various running conditions. A new system of measurement using two differential variable reluctance transducers (DVRT) was developed. It is evident that the roller skew of the double row tapered roller bearing can be measured. The shaft rotational speed has a significant effect on roller skew but the radial load has little effect.
\end{abstract}

\section{Introduction}

Roller skew is mainly generated from the tangential friction force on the rib-roller end contact. Many studies have been conducted to look into the effects of different static and dynamic parameters of the bearing elements. The tapered roller bearing has had its share of these studies. Zhou and Hoeprich [1] presented a model for predicting tapered roller bearing torque in their study. The analytical result was compared with test data and was concluded to be in good agreement. S.Cretu $e t$ al. [2-3] developed a quasi-dynamic model for tapered roller bearings which was used to analyse various parameters such as sliding velocity and film thickness at various interactions that exist with the tapered roller bearing envelope. Yang et al. [4] measured roller skew in a tapered roller bearing using non-vibrating CPD probe. The study looked at roller skew value when the bearing was lubricated with different lubricants and run at various shaft speeds. The result obtained in this study was compared with the roller skew obtained in a quasi-static analytical model developed in work published by Nelias et al. [5]. The model was used to analyse a single row tapered roller bearing. However, these investigations on roller skew are limited in a single row tapered roller bearing [4-7]. There is little information in either measurement or modelling of the roller skew in a double row tapered bearing. Measurement of the roller skew in a double row tapered bearing is more difficult because it requires access to the roller surface precisely during bearing operation.

This paper presents the results of the study to measure roller skew in a double row tapered roller bearing under various load and speed conditions with a newly developed system of measurement.

\section{Experimental Apparatus and Procedure}

A newly developed system of measurement is composed of two differential variable reluctance transducers (DVRT), MicroStrain DEMOD-DVRT, Agilent U2542A DAQ and Agilent Measurement manager software package, Honeywell S\&C Hall Effect and spectrum analyser (hp 3582A Spectrum Analyzer). A double row tapered roller bearing (Cooper's Heavy Duty Tapered roller bearing) was used and its details are shown in Table 1.

As shown in Fig.1, the two DVRT transducers were placed at the opposite ends of the roller 10mm apart. They were incorporated into the bearing envelope such that they were placed above the rollers and perpendicular to the outer race contact plane with a minimum clearance of $0.1 \mathrm{~mm}$. The test bearing was mounted on a test rig on which a radial and thrust load can be applied. At a constant radial 
load of $6.4 \mathrm{kN}$, thrust load of $0 \mathrm{kN}, 6.4 \mathrm{kN}$ and $12.4 \mathrm{kN}$ were centrically applied to the test bearing. The bearing was run, for each of these combined loads, at the shaft rotational speeds of $1000 \mathrm{rpm}$, 2000rpm, $2500 \mathrm{rpm}, 3000 \mathrm{rpm}$ respectively. For each of these speeds, the shaft was run both forward and reverse. The shaft speed was measured and monitored using Honeywell S\&C Hall Effect and hp 3582A Spectrum Analyzer. The temperature of the bearing was also monitored to prevent bearing over-heating. Both the radial and thrust load were measured and monitored by load cells mounted on the test ring.

Table 1 Dimension of a sample bearing

\begin{tabular}{|l|l|l|l|l|l|l|}
\hline $\begin{array}{l}\text { Roller } \\
\text { Length }\end{array}$ & $\begin{array}{l}\text { Roller } \\
\text { Angle }\end{array}$ & $\begin{array}{l}\text { Cone (inner race) } \\
\text { Contact Angle }\end{array}$ & $\begin{array}{l}\text { Number of } \\
\text { Rollers }\end{array}$ & $\begin{array}{l}\text { Roller End } \\
\text { Diameter }\end{array}$ & $\begin{array}{l}\text { Bearing Bore } \\
\text { Diameter }\end{array}$ & $\begin{array}{l}\text { Pitch } \\
\text { Diameter }\end{array}$ \\
\hline $18.633[\mathrm{~mm}]$ & $8^{\circ}$ & $26.5^{\circ}$ & 32 & $13.83[\mathrm{~mm}]$ & $60[\mathrm{~mm}]$ & $92[\mathrm{~mm}]$ \\
\hline
\end{tabular}

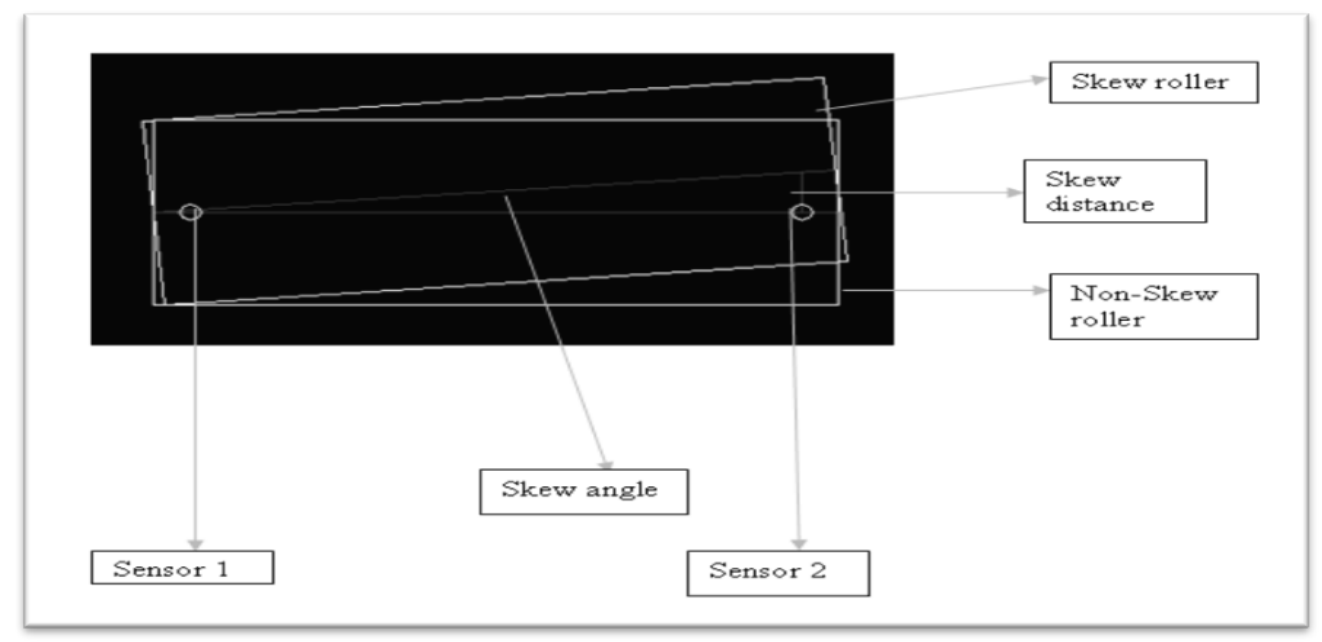

Fig.1 Sketch diagram of roller skew

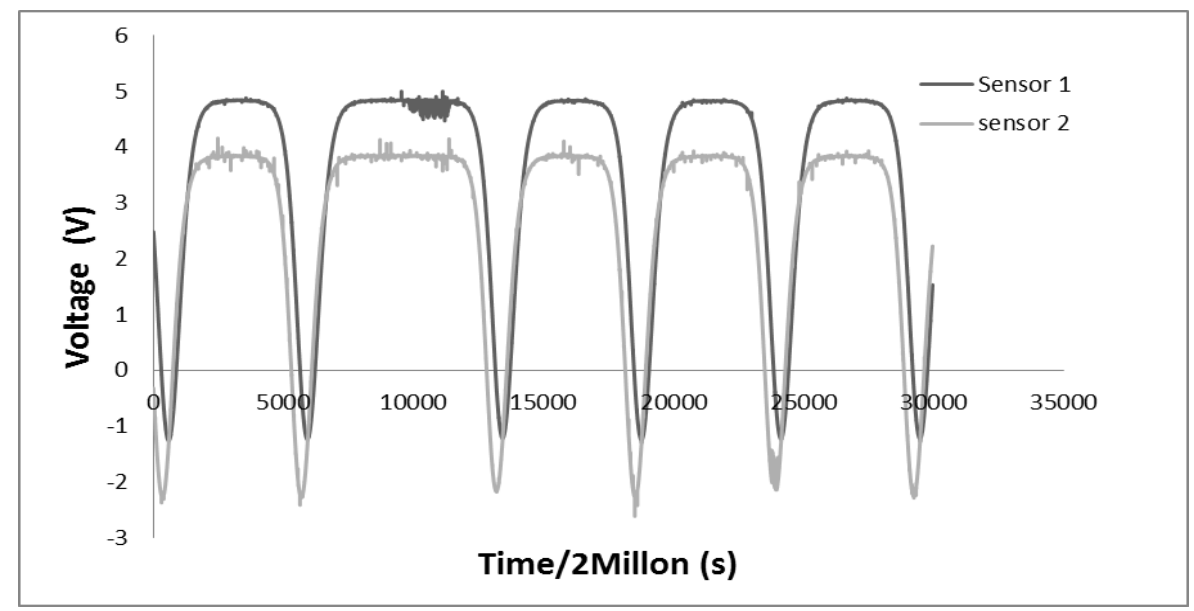

Fig.2 Signal from the two transducers

The signal from the transducers was conditioned using MicroStrain DEMOD-DVRT and acquired using Agilent U2542A DAQ and Agilent Measurement manager software at a sampling rate of 2Ms/s. At this sampling rate, the minimum roller skew that can be measured at the maximum shaft speed in which this experiment was conducted is $0.019^{\circ}$. The resolution of the measured skew is dependent on roller velocity and sampling rate. Data from the transducer were collected at each running condition, 
when the shaft speed was stable and bearing thermal equilibrium had been reached. Data were collected over an average of $6 \mathrm{~s}$.

The acquired signal was filtered to remove noise and analysed for phase difference. This was done by using the point of closest approach on the roller which corresponds to the minimum signal from the transducers as shown in Fig.2. The time at which this occurs for both signals was taken sequentially and subtracted from each other to obtain the phase difference. This was used to determine the average roller skew. Roller skew was measured at two locations. Position 1 was $15^{\circ}$ from most loaded roller while position 2 was $90^{\circ}$ from position 1 .

\section{Results and Discussion}

Figs.3-4 show the results of the average roller skew under various loading and speed conditions. It can be seen that the average roller skew increases with an increase of shaft speed at all loading conditions and at both measurement positions. The rate of increase with respect to shaft speed is approximately constant.

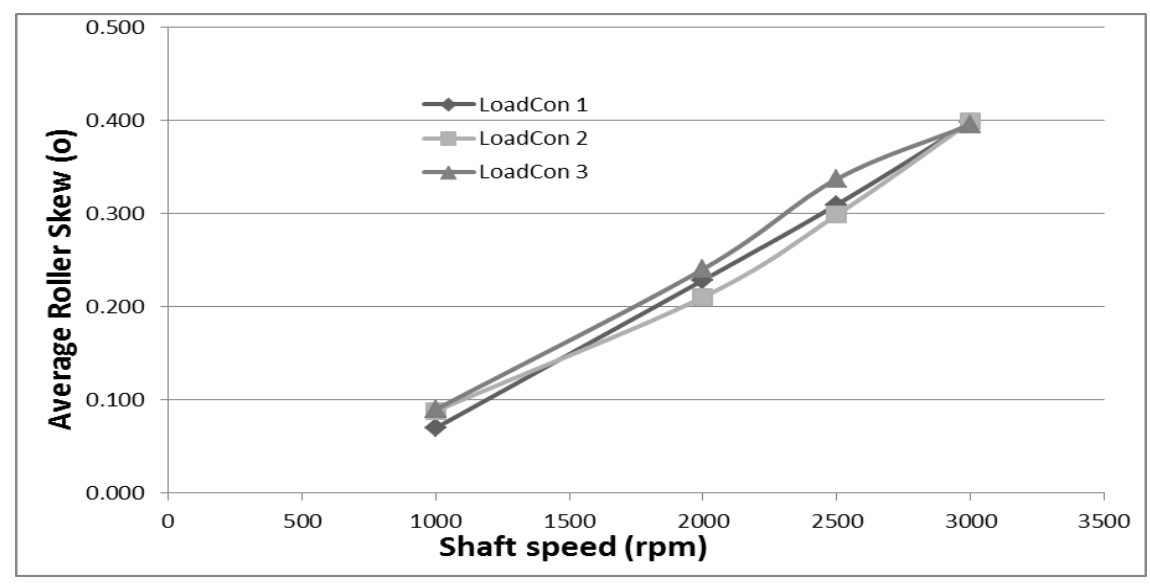

Fig.3 Average roller skew against shaft speed at position 1 (Loading Condition 1: 0-6.4kN; Loading Condition 2: 6.4kN \& Loading Condition 3: $6.4-12.4 \mathrm{kN}$ )

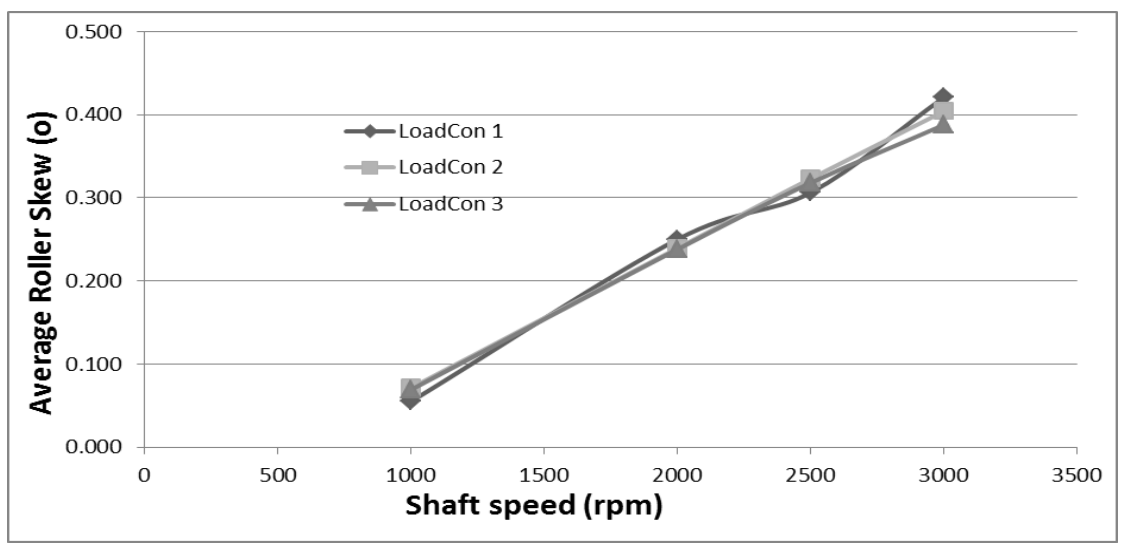

Fig.4 Average roller skew against shaft speed at position 2 (Loading Condition 1: 0-6.4kN; Loading Condition 2: 6.4kN \& Loading Condition 3: $6.4-12.4 \mathrm{kN})$

It was observed that under the same shaft speed in position 1, the roller skew was higher in loading condition 3 compared with the other two loading conditions. This was statistically significant 
$(\mathrm{p}<0.05)$ at 2000rpm and 2500rpm as shown in Fig.3. This was also the case when loading conditions 1-2 were compared. Similarly at position 2, comparing the skew observed at different load conditions, all but condition 2 compared with loading condition 3 at 1000rpm and 2000rpm were statistically significant $(\mathrm{p}<0.05)$ (Figure 4).

As shown in Figs.3-4, the roller skew at both positions increases with shaft speeds. The differences seen at these positions under the same load condition and running speed were statistically significant ( $\mathrm{p}<0.05)$. This however was not the case at load condition 3, 1000rpm and 2000rpm. These were found not to be significant. Additionally, the difference seen in roller skew at different shaft speed was much larger compared with differences seen under different load conditions and at different positions.

Under all conditions in which the test bearing was subjected to, the roller skew of a double row tapered roller bearing was observed. It ranges from $0.07^{\circ}$ to $0.42^{\circ}$ depending on running conditions. These values were comparable to the findings in the previous work [4]. It is evident that shaft speed has more effect on roller skew compared with applied load. With an increase in thrust load while radial load was kept constant, the load acting at the roller/race and roller/flange will increase. As expected, the friction force at this contact will increase. However, skew moment is resisted by the 'restoring moment' at the roller/race contact and 'blocking moment' at the roller/flange contact. These moments are function of the normal load acting at these contacts and the orientation of the roller.

\section{Conclusions}

The roller skew in a double row tapered roller bearing can be measured with a newly developed system of measurement using two DVRTs. It is found that roller skew changes when the running condition changes. The shaft speed plays a great role in the roller skew of a double row tapered roller bearing. The roller skew of the double row tapered roller bearing is not sensitive to the radial loading.

\section{Acknowledgements}

This work was financially supported by the KTP program from UK TSB and Cooper Roller Bearings Company Ltd.

\section{References}

[1] R.S. Zhou, and M.R. Hoeprich, Torque of tapered roller-bearings, ASME J. Tribology. 113 (3) (1991) 590-597.

[2] S. Cretu, I. Bercea and N. Mitu, A dynamic analysis of tapered roller bearing under fully flooded conditions, Part 1: Theoretical formulation, Wear. 188 (1-2) (1995) 1-10.

[3] S. Cretu, N. Mitu, and I. Bercea, A dynamic analysis of tapered roller-bearings under fully flooded conditions, Part 2: Results, Wear. 188(1-2) (1995) 11-18.

[4] Y.Y. Yang, S. Danyluk, and W. Hoeprich, A study on rolling element skew measurement in a tapered roller bearing with a specialized capacitance probe, ASME J. Tribology. 122 (3) (2000) 534-538.

[5] D. Nelias, I. Bercea, and V. Paleu, Prediction of roller skewing in tapered roller bearings, Tribology Transactions. 51 (2) (2008) 128-139.

[6] T.A. Harris, Rolling Bearing Analysis $4^{\text {th }}$ edition, 2001(John Wiley \& sons, Inc. New York).

[7] W. Wang, P.L. Wong and Z. Zhang, Partial EHL analysis of rib-roller end contact in tapered roller bearings, Tribology International. 29 (4) (1996) 313-321. 